\title{
TXS 2116-077: A Gamma-Ray Emitting Relativistic Jet Hosted in a Galaxy Merger
}

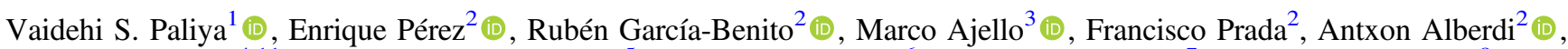 \\ Hyewon Suh $^{4,11}$ (D) C. H. Ishwara Chandra ${ }^{5}$, Alberto Domínguez ${ }^{6}$ (iD), Stefano Marchesi ${ }^{7}$ (D), Tiziana Di Matteo ${ }^{8}$, \\ Dieter Hartmann $^{3}$ (D), and Marco Chiaberge ${ }^{9,10}$ (D) \\ ${ }^{1}$ Deutsches Elektronen Synchrotron DESY, Platanenallee 6, D-15738 Zeuthen, Germany; vaidehi.s.paliya@gmail.com \\ ${ }^{2}$ Instituto de Astrofísica de Andalucía (CSIC), E-18008, Granada, Spain \\ ${ }^{3}$ Department of Physics and Astronomy, Clemson University, Kinard Lab of Physics, Clemson, SC 29634-0978, USA \\ ${ }^{4}$ Subaru Telescope, National Astronomical Observatory of Japan (NAOJ), 650 North A'ohoku Place, Hilo, HI 96720, USA \\ ${ }^{5}$ National Centre for Radio Astrophysics, TIFR, Post Bag 3, Ganeshkhind, Pune 411007, India \\ ${ }^{6}$ IPARCOS and Department of EMFTEL, Universidad Complutense de Madrid, E-28040 Madrid, Spain \\ ${ }^{7}$ INAF-Osservatorio di Astrofisica e Scienza dello Spazio di Bologna, Via Piero Gobetti, 93/3, I-40129, Bologna, Italy \\ ${ }^{8}$ McWilliams Center for Cosmology, Physics Department, Carnegie Mellon University, Pittsburgh, PA 15213, USA \\ ${ }^{9}$ Space Telescope Science Institute, 3700 San Martin Drive, Baltimore, MD 21218, USA \\ ${ }^{10}$ Johns Hopkins University-Center for Astrophysical Sciences, 3400 North Charles Street, Baltimore, MD 21218, USA \\ Received 2020 January 29; revised 2020 February 6; accepted 2020 February 10; published 2020 April 7
}

\begin{abstract}
What triggers collimated relativistic outflows or jets from the centers of galaxies remains a fundamental question in astrophysics. The merging of two galaxies has been proposed to realize the conditions to successfully launch and drive such jets into the intergalactic medium. However, evidence for the operation of this mechanism is scarce. Here we report the first unambiguous detection of an ongoing merger of a narrow-line Seyfert 1 galaxy, TXS 2116 -077 , hosting a closely aligned, $\gamma$-ray emitting relativistic jet with a Seyfert 2 galaxy at a separation of $\sim 12 \mathrm{kpc}$, using the observations taken with the $8.2 \mathrm{~m}$ Subaru Telescope. Our subsequent follow-up observations with the $10.4 \mathrm{~m}$ Gran Telescopio Canarias, $4.2 \mathrm{~m}$ William Herschel Telescope, and Chandra X-ray observatory have provided what is likely to be the first glimpse of the merging environment hosting a closely aligned relativistic jet. Our finding that the jet is considerably younger than the merger demonstrates that jet activity can be triggered by galaxy mergers and that $\gamma$-ray detected narrow-line Seyfert 1 galaxies represent the beginning phase of that activity. These results also highlight the crucial role of mergers in shaping the fate of galaxies in their cosmological evolution and are consistent with recent studies focused on the host galaxy imaging of this enigmatic class of active galactic nuclei.
\end{abstract}

Unified Astronomy Thesaurus concepts: AGN host galaxies (2017); Galaxy interactions (600); Galaxy jets (601); Relativistic jets (1390); Blazars (164)

\section{Introduction}

It is now well established that essentially every large galaxy, including our own Milky Way, hosts a massive black hole at its center (e.g., Kormendy \& Richstone 1995). One in a hundred galaxies displays an active galactic nucleus (AGN) where the emission from the nuclear region can outshine the galaxy itself, and controls its growth via strong radiative feedback (see Fabian 2012). This emission is due to the black hole accreting circumnuclear gas, but the circumstances that bring the gas near the region of influence of the black hole are still unclear. Among all AGN, 15\% exhibit spectacular, often large-scale, bipolar relativistic jets (Kellermann et al. 1989) where particles are confined by the magnetic field and accelerated to near the speed of light. AGN with jets aligned with our line of sight are called blazars and exhibit highly variable emission across the entire electromagnetic spectrum. Blazars are the most powerful, persistent sources of radiation in the universe (e.g., Ghisellini et al. 2014; Paliya et al. 2019a).

Jetted AGN, including blazars, are usually hosted in massive, old elliptical galaxies (see, e.g., Laor 2000; Chiaberge \& Marconi 2011) which are likely to be formed in major merging events (e.g., Toomre \& Toomre 1972). Thus, it is surmised that galaxy mergers are responsible for the launch of relativistic jets (Wilson \& Colbert 1995; Floyd et al. 2008;

\footnotetext{
${ }^{11}$ Subaru Fellow.
}

Ramos Almeida et al. 2013; Chiaberge et al. 2015). However, these studies were focused mostly on quasars with off-axis jets, i.e., sources viewed at large angles with respect to jet axis $\left(\gtrsim 30^{\circ}\right)$. Moreover, quasars studied in previous works were found to reside in already evolved ellipticals or exhibiting morphologies as disturbed as those expected from a late stage merger (Chiaberge et al. 2015). In blazars, on the other hand, the on-axis viewing geometry makes study of the host galaxy extremely difficult due to Doppler-boosted, bright jet emission and results acquired from various campaigns focusing on the host galaxy properties of blazars remained inconclusive, albeit providing hints in support of the findings derived by studying off-axis jets (e.g., Kotilainen et al. 1998; Nilsson et al. 2003; Olguín-Iglesias et al. 2016). The missing link, however, is to find examples of closely aligned jet systems caught in the act of merging and/or hosted in late-type (i.e., young) AGN, which can reveal the stage in the AGN evolution when the jet activity was recently triggered.

Among all classes of AGN, $\gamma$-ray detected narrow-line Seyfert 1 ( $\gamma$-NLSy1) galaxies are probably the optimal systems as they are known to host closely aligned, low-power relativistic jets (Abdo et al. 2009; Foschini et al. 2015; Paliya et al. 2019b) and are thus probably the best systems for exploring the jet-host-galaxy connection. NLSy1 galaxies are classified based on their optical spectral characteristics, such as broad permitted lines with small widths $(\mathrm{H} \beta$ 
FWHM $<2000 \mathrm{~km} \mathrm{~s}^{-1}$ ), weak [O III] emission ([O III] $/ \mathrm{H} \beta$ flux ratio $<3$ ), and strong $\mathrm{Fe}$ complexes (Osterbrock \& Pogge 1985; Goodrich 1989). The detection of variable $\gamma$-ray emission from about half-a-dozen radio-loud NLSy1 objects with the Fermi Large Area Telescope (e.g., Abdo et al. 2009) has provided the strongest evidence in support of the relativistic beaming. These enigmatic sources, in general, are thought to be rapidly accreting (Boroson \& Green 1992), low-luminosity AGNs in the early phase of their evolution (Mathur 2000; Grupe \& Mathur 2004; Berton et al. 2018). They have also been found to reside in spiral/late-type galaxies (Kotilainen et al. 2016; Berton et al. 2019; Olguín-Iglesias et al. 2020) providing supportive evidence about their young nature.

The facts that make $\gamma$-NLSy1s the most promising systems to study the jet launching processes are their relatively low jet powers and young nature (e.g., Foschini et al. 2015). A low jet power indicates that the underlying host galaxy properties can be explored in a much better fashion than is possible for more powerful blazars. Furthermore, studying a young $\gamma$-NLSy 1 also provides an opportunity to reveal the jet-host galaxy interaction when the system is rapidly growing and possibly hosts a nascent jet.

Here we report observations of TXS 2116-077, a NLSy1 galaxy (Rakshit et al. 2017) with redshift $z=0.26$ (corresponding to a distance of $1329 \mathrm{Mpc}$; Planck Collaboration et al. 2016). TXS 2116-077 possesses a relativistic jet closely aligned to our line of sight as confirmed by the detection of its variable $\gamma$-ray flux, the observation of a compact radio core at $8.4 \mathrm{GHz}$, its large radio-loudness, and flat radio and X-ray spectra (Paliya et al. 2018; Yang et al. 2018). The broadband spectral energy distribution of TXS 2116-077 resembles that of blazars (Yang et al. 2018; Paliya et al. 2019b), which again confirms the presence of a closely aligned relativistic jet in this NLSy1 galaxy. Furthermore, the low-resolution Sloan Digital Sky Survey (SDSS) optical image of this source exhibits an extended structure, which was tentatively explained as possibly due to merger or enhanced star-forming activities (Paliya et al. 2018; Yang et al. 2018).

We have carried out, for the first time, a high-resolution, adaptive optics (AO) enabled, $J$-band imaging of the host galaxy of TXS 2116-077 with the $8.2 \mathrm{~m}$ Subaru Telescope, revealing two galaxies in the act of merging. We have followed it up with long-slit optical spectroscopy and integral-field unit (IFU) observation with $10.4 \mathrm{~m}$ Gran Telescopio Canarias (GTC) and $4.2 \mathrm{~m}$ William Herschel Telescope (WHT), and a $10 \mathrm{ksec}$ Chandra X-ray observatory pointing to study the host galaxy environment of a beamed AGN in an unprecedented detail. In Section 2, we briefly describe the adopted data reduction procedure and present the acquired results in Section 3. We discuss our findings in Section 4 and summarize in Section 5.

\section{Data Reduction and Analysis}

\subsection{Host Galaxy Imaging with Subaru}

We obtained a high-resolution $J$-band image of TXS 2116 -077 with the InfraRed Camera and Spectrograph (IRCS; Kobayashi et al. 2000), in combination with its AO system AO 188 (Hayano et al. 2010), mounted at the Subaru Telescope on 2018 June 16. We used a resolution of 0 ." 052 per pixel for a corresponding 54" field of view. The Laser Guide Star mode was used for $\mathrm{AO}$ and observations were performed in imaging mode. The total integration time was 34 minutes while accumulating 17 frames with an exposure time of $120 \mathrm{~s}$ per frame using a 5 point dither pattern. The natural seeing was $\sim 0$." 7 and the AO-corrected seeing was 0 ". 2 .

The IRCS data was analyzed with Image Reduction and Analysis Facility (IRAF; Tody 1986). This includes the creation of a bad pixel mask, background subtraction, flatfielding estimate of the dither offsets, and final stacking of the data. A nearby star, SDSS J211853.33-073214.3 (R.A.: $21^{\mathrm{h}} 18^{\mathrm{m}} 53.33$, decl.: $\left.-07^{\circ} 32^{\prime} 14{ }^{\prime \prime} 34\right)$, located $\sim 14^{\prime \prime}$ northeast of the $\gamma$-NLSy1 was used as a reference for the flux calibration.

\subsection{Long-slit Optical Spectroscopy}

ISIS at WHT. Long-slit spectrophotometric observations of TXS 2116-077 were obtained in service time with the Intermediate-dispersion Spectrograph and Imaging System (ISIS) double-arm spectrograph mounted on WHT of the Isaac Newton Group at the Roque de los Muchachos Observatory on the Spanish island of La Palma. They were acquired on 2018 August 13 with an average seeing of 0.9 . To cover both nuclei detected in the Subaru image, we used a slit position angle of 116.7. Grating R158B was used in the blue arm centered at $4500 \AA$ with a wavelength range between 3370 and $5400 \AA$ and $1.45 \AA$ pixel $^{-1}$ dispersion. In the red arm, grating R158R centered in $7500 \AA$ covered between 5300 and $10000 \AA$ with a dispersion of $1.82 \AA$ pixel $^{-1}$. The slit width was 1 !' 2 . A total of six exposures of $1800 \mathrm{~s}$ each were taken simultaneously in each arm. The reduction was performed using IRAF routines in the usual manner. For both arms, the standard star G191-B2B was used to produce final sky subtracted and flux calibrated spectra.

OSIRIS at GTC. Long-slit spectrophotometric observations were obtained under the Director's Discretionary Time (DDT) program using the Optical System for Imaging and lowIntermediate-Resolution Integrated Spectroscopy (OSIRIS) spectrograph mounted at GTC located at the Roque de los Muchachos Observatory on the island of La Palma, Spain. They were taken on 2018 August 16 with an average seeing of 0 ". 9 during dark time using grating R2000B and a slit width of 0 ". 8 . The wavelength range covered between 3950 and $5650 \AA$ at a dispersion of $0.86 \AA_{\text {pixel }}^{-1}$. A total of four exposures of $1800 \mathrm{~s}$ each were taken. The reduction was performed using IRAF routines following the standard procedures. The standard star Ross 640 was used to obtain the sensitivity curve and perform the flux calibration.

\subsection{IFU Observations}

Multi-Espectrógrafo en GTC de Alta Resolución para Astronomía (MEGARA) is an optical IFU and multi-object spectrograph installed at the GTC (Gil de Paz et al. 2016). The MEGARA IFU encompasses 567 contiguous hexagonal fibers, each with a long diagonal of 0 ". 62 , resulting in a $12.5 \times 11.3$ $\operatorname{arcsec}^{2}$ field of view in the shape of a rectangle. Sky observations are obtained simultaneously with eight fiber bundles with seven dedicated hexagonal fibers each located at the outermost parts of the field of view $(R>1.5)$. MEGARA IFU spectra of TXS 2116-077 were obtained under DDT program on the night of 2018 October 1 using the LR-I VPH grating that covers from $\sim 7200$ to $8650 \AA$ with a reciprocal linear dispersion of $0.37 \AA$ pixel $^{-1}$. We obtained six exposures of $1690 \mathrm{~s}$ each. The seeing conditions were around 0 ". 6 . To flux-calibrate the LR-I spectra, several exposures of 
the flux standard star HR 7950 were obtained under similar airmass and seeing conditions. We also obtained calibration data, including ThNe and ThAr arc lamps and halogen lamps.

The raw IFU spectra were processed using the MEGARA data reduction pipeline ${ }^{12}$ (MDRP; S. Pascual 2020, in preparation). The main steps include: bias correction, trimming, identification of the position of the spectra on the detector along the dispersion axis, model mapping and extraction of each individual spectrum, fiber-flat correction, wavelength calibration, flux calibration, and sky subtraction. The final products of the MDRP are row-stacked spectra (RSS) 2D images containing 623 fiber spectra. The $\mathrm{H} \alpha$ line shows a second, broad component in some spaxels around the Seyfert 1 nucleus. Thus, we used a multi-component Gaussian model to fit the [N II] + $\mathrm{H} \alpha$ window. For the sake of visualization, we have transformed the hexagonal spaxel grid of the RSS data into a square pixel shape grid of 0 ". 25 per pixel.

\subsection{Very Large Array Observations}

The archival Very Large Array (VLA) data of TXS 2116 -077 at $8.4 \mathrm{GHz}$, observed on 1998 May 18, was analyzed using the Astronomical Image Processing System (AIPS), following standard procedures. The flux scale was set using the standard flux calibrator 3C 286. The flux and phase calibrations were transferred to the target via the secondary calibrator. A few rounds of self-calibration were performed to correct for phase variations, which improved the image quality. The resolution of the final image is 0 ". $32 \times 0$ ". 23 at a position angle of $-14^{\circ}$. The root mean square (rms) noise floor of the map is $42 \mu \mathrm{Jy}$ beam $^{-1}$. The $\gamma$-NLSy1 remained unresolved at this frequency and resolution. We used the Gaussian fitting program JMFIT in AIPS to get the flux density. The peak and integrated flux density is nearly identical confirming the compact nature of the source. The integrated flux density of the target is $91.87 \mathrm{mJy}$. To compute the error on the flux, we have added the calibration error, Gaussian fitting error, and the rms noise of the map in quadrature, giving the final error as $0.17 \mathrm{mJy}$. The companion Seyfert 2 galaxy, on the other hand, remain undetected and we derived the $3 \sigma$ flux upper limit as $0.13 \mathrm{mJy}$.

A deep radio imaging of TXS 2116-077 with Very Large Baseline Array (VLBA) has recently been performed on 2018 March 30. We analyzed this publicly available VLBA observation at $6 \mathrm{~cm}(5 \mathrm{GHz})$. A sustained data-recording rate of $512 \mathrm{Mbits} \mathrm{s}^{-1}$ in two-bit sampling was used. The frequency band was split into four intermediate frequencies (IFs) of 64 $\mathrm{MHz}$ bandwidth each, for a total synthesized bandwidth of 256 MHz. Each IF was in turn split into 256 channels of $250 \mathrm{KHz}$ bandwidth. The data were corrected for Earth-orientation and ionospheric effects. The source BL Lac was used as bandpass calibrator. The data were correlated at the NRAO data processor using an averaging time of $2 \mathrm{~s}$. We performed standard a-priori gain calibration using the measured gains and system temperatures of each antenna. This calibration, as well as the data inspection and editing, were done within AIPS. The flux scale was set using the calibrator BL Lac. The observation resulted in a beam of $5.3 \times 2.4$ millarcsec $^{2}$, at a PA of $-2^{\circ}$. A compact radio emission positionally consistent with the $\gamma$ NLSy1 galaxy is found with no hints of any extended features or knots. The peak flux maximum is located at the position the

\footnotetext{
$\overline{12 \text { https://github.com/guaix-ucm/megaradrp }}$
}

R.A.: $21^{\mathrm{h}} 18^{\mathrm{m}} 52^{\mathrm{s}} .96$ and decl., $\mathrm{J} 2000=-07^{\circ} 32^{\prime} 27^{\prime \prime} .58$, with a flux density of $27.93 \pm 0.23 \mathrm{mJy} \mathrm{b}^{-1}$. There is no detection of the companion Seyfert 2 galaxy, with an upper limit of 0.13 mJy beam $^{-1}$.

\subsection{Chandra Observations}

Chandra X-ray observatory carried out a DDT observation of TXS 2116-077 on 2018 September 15 for a net exposure of $10 \mathrm{ksec}$. We used the nominal aim point of the Advanced CCD Imaging Spectrometer chip (ACIS-S3) and the data were collected in a full readout mode resulting in $3.241 \mathrm{~s}$ readout time. The data were reprocessed using chandra_repro and an exposure-corrected image was generated using fluximage. We scanned the Chandra image with the source detection algorithm wavedetect to search for X-ray emitting objects. A bright X-ray source (R.A., J2000 $=21^{\mathrm{h}} 18^{\mathrm{m}} 52^{\mathrm{s}} .95$ and decl., $\mathbf{J} 2000=-07^{\circ} 32^{\prime} 27^{\prime \prime} 54$ ) coincident with the $\gamma$-NLSy 1 is identified with the separation between the X-ray and radio positions as 0 !' 19 . The excellent spatial resolution of Chandra $(\sim 0$ ".5) allowed us to resolve the merging system, however the companion Seyfert 2 galaxy remains below the detection limit.

We used the task specextract to extract the source and background spectra and corresponding ancillary and response matrix files. A source region of 1 ". 5 centered at the $\gamma$-NLSy 1 galaxy was adopted and we selected the background as a circle of $10^{\prime \prime}$ from a nearby source-free region. We rebinned the source spectrum to have 1 count per bin and performed the fitting in the energy range of $0.5-7 \mathrm{keV}$ using C-statistics (Cash 1979) in XSPEC (Arnaud 1996).

\section{Results}

We show the high-resolution, AO enabled, $J$-band image of TXS 2116-077 in Figure 1 and overplot the $8.4 \mathrm{GHz}$ VLA contours, which suggests the presence of a compact radio jet associated with the $\gamma$-NLSyl galaxy. As can be seen, two galactic nuclei are resolved (separation $\sim 12 \mathrm{kpc}$ ) and appear to be interacting. This merging phenomenon is confirmed from the long-slit optical spectroscopy which reveals both objects to be located at the same redshift, as shown in Figure 2. The optical spectrum of the companion galaxy shows emission lines, thus hosting an AGN, which, in the absence of broad emission lines, can be classified as a Seyfert 2.

The $\mathrm{H} \alpha$ flux map acquired from the integral-field spectroscopy unit MEGARA mounted on the GTC shows two strong peaks, spatially coincident with both nuclei (Figure 3 ). The $\gamma-$ NLSy 1 nucleus is surrounded by a $\sim 2^{\prime \prime}$ ring-like distribution of $\mathrm{H} \alpha$ emitting material and a curved filament connects both nuclei and extends to the south of the $\gamma$-NLSy1. The $\mathrm{H} \alpha$ velocity and velocity dispersion maps, as shown in the bottom panel of Figure 3, reveal complex kinematic structures within the merging system. There is no clear large-scale systematic rotation pattern, with patchy blue- and redshifted regions (relative to the $\gamma$-NLSy1 nucleus). This observation is consistent with the merging driven turbulence, possibly due to merger induced shocks, the presence of the relativistic jet, and their interaction. Interestingly, there is a fast outflowing region detected at $\sim 1^{\prime \prime}$ northeast of the $\gamma$-NLSy1 nucleus, possibly hinting at a buried or nascent jet (Hopkins \& Elvis 2010). 


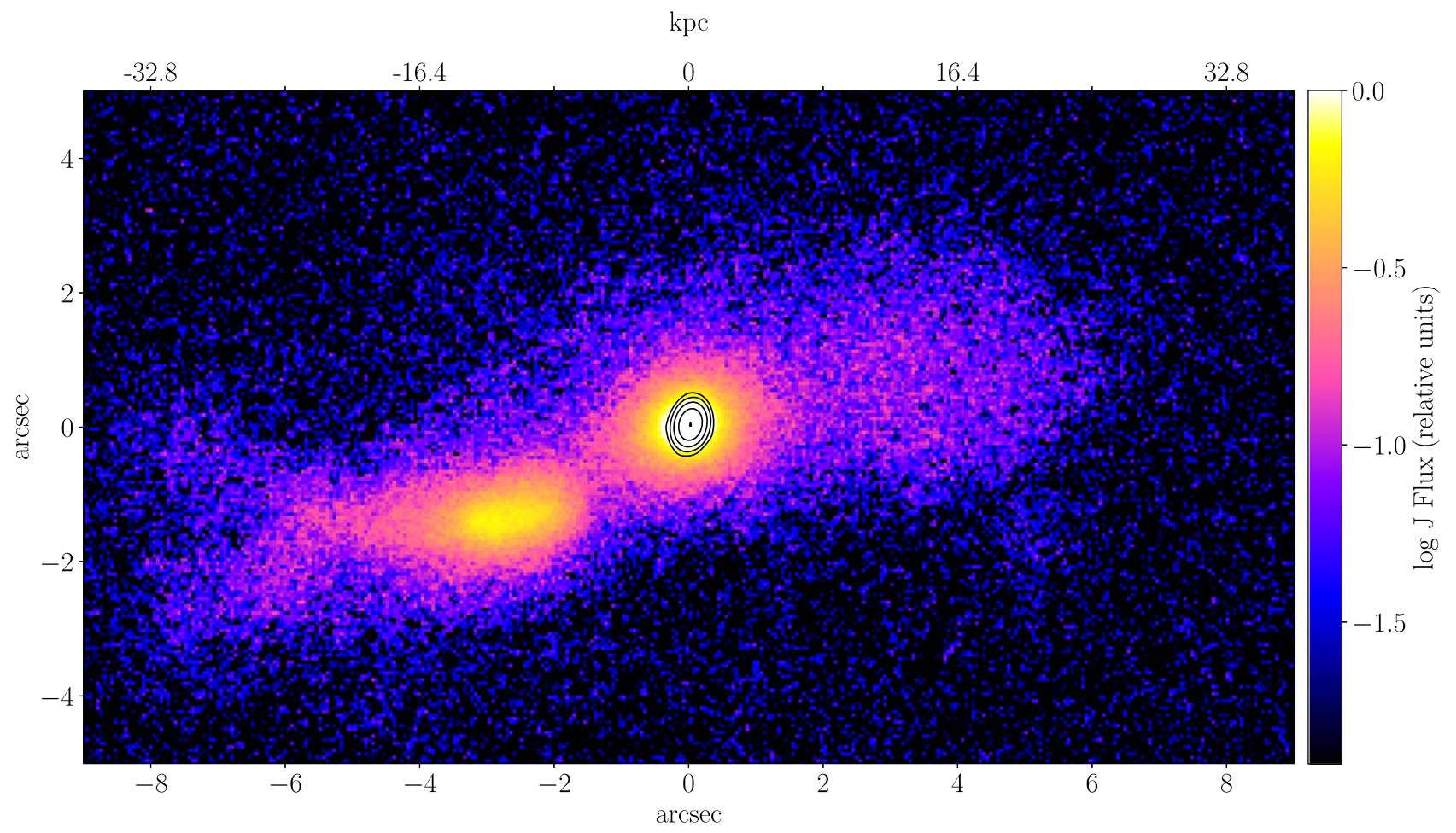

Figure 1. Adaptive optics enabled $J$-band image of TXS 2116-077 $(z=0.26)$ obtained with IRCS mounted at the Subaru Telescope. The image is centered at the $\gamma$ NLSy1 galaxy. The colorbar represents the relative flux on a logarithmic scale. The image scale is 0. " 052 per pixel. Overplotted are $8.4 \mathrm{GHz}$ VLA contours (levels from $0.2 \mathrm{mJy}_{\text {beam }}^{-1}$, i.e., five times the off-source rms, to $89.7 \mathrm{mJy} \mathrm{beam}^{-1}$ in five logarithmic intervals). The beam size is $0{ }^{\prime \prime} 32 \times 0$.' 23 with a position angle of $-13^{\circ} .81$. Top axis shows the scale in kpc with $1^{\prime \prime}=4.06 \mathrm{kpc}$. North is up and east to the left.

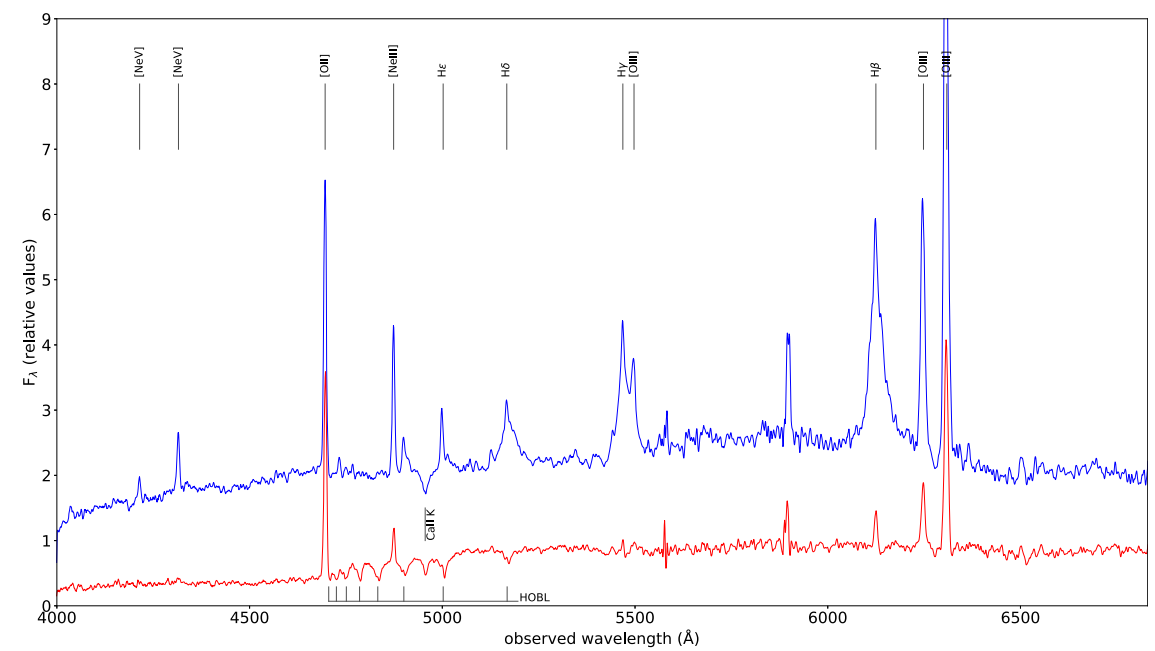

Figure 2. Integrated long-slit spectra of the two galaxies, the $\gamma$-NLSy1 (blue) and the Seyfert 2 (red), taken with ISIS at WHT and OSIRIS at GTC. The main emission and absorption lines are labeled, including the higher order Balmer lines in the Seyfert 2.

The high-resolution, $0.5-7 \mathrm{keV}$ image of the merging system TXS 2116-077 is shown in Figure 4 and we overlaid the Subaru contours to guide the eyes. Only the $\gamma$-NLSy1 galaxy is detected, whose spectrum is well-fitted (C-statistics $=146.1$ for 166 degree of freedom) by a simple power law corrected for Galactic absorption $\left(N_{\mathrm{H}}=6.99 \times 10^{20} \mathrm{~cm}^{-2}\right.$; Kalberla et al. 2005). The best-fit photon index is $\Gamma=1.64_{-0.18}^{+0.19}$, and the $0.5-7 \mathrm{keV}$ unabsorbed energy flux is $f_{0.5-7}=4.27_{-0.39}^{+0.49} \times 10^{-13} \mathrm{erg} \mathrm{cm}^{-2} \mathrm{~s}^{-1}$. Such a flat X-ray spectrum provides a supportive evidence of the jet based origin of the observed X-ray emission (see also Paliya et al. 2019b). Furthermore, the corresponding $K$-corrected, isotropic luminosity is $L_{0.5-7}=8.71_{-0.95}^{+1.03} \times 10^{43} \mathrm{erg} \mathrm{s}^{-1}$. For the Seyfert 2 galaxy, we derived the $3 \sigma$ flux upper limit, $f_{0.5-7}<$ $3.98 \times 10^{-16} \mathrm{erg} \mathrm{cm}^{-2} \mathrm{~s}^{-1}\left(L_{0.5-7}<8.80 \times 10^{40} \mathrm{erg} \mathrm{s}^{-1}\right)$, assuming a typical photon index $\Gamma=2$ and a line-of-sight column density as that of $\gamma$-NLSy1. If the source is a Compton thick AGN (with intrinsic $N_{\mathrm{H}}=1 \times 10^{24} \mathrm{~cm}^{-2}$ ), the $3 \sigma$ unabsorbed flux upper limit is instead $f_{0.5-7}<2.78 \times 10^{-14}$

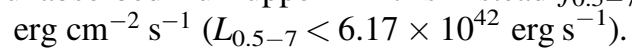



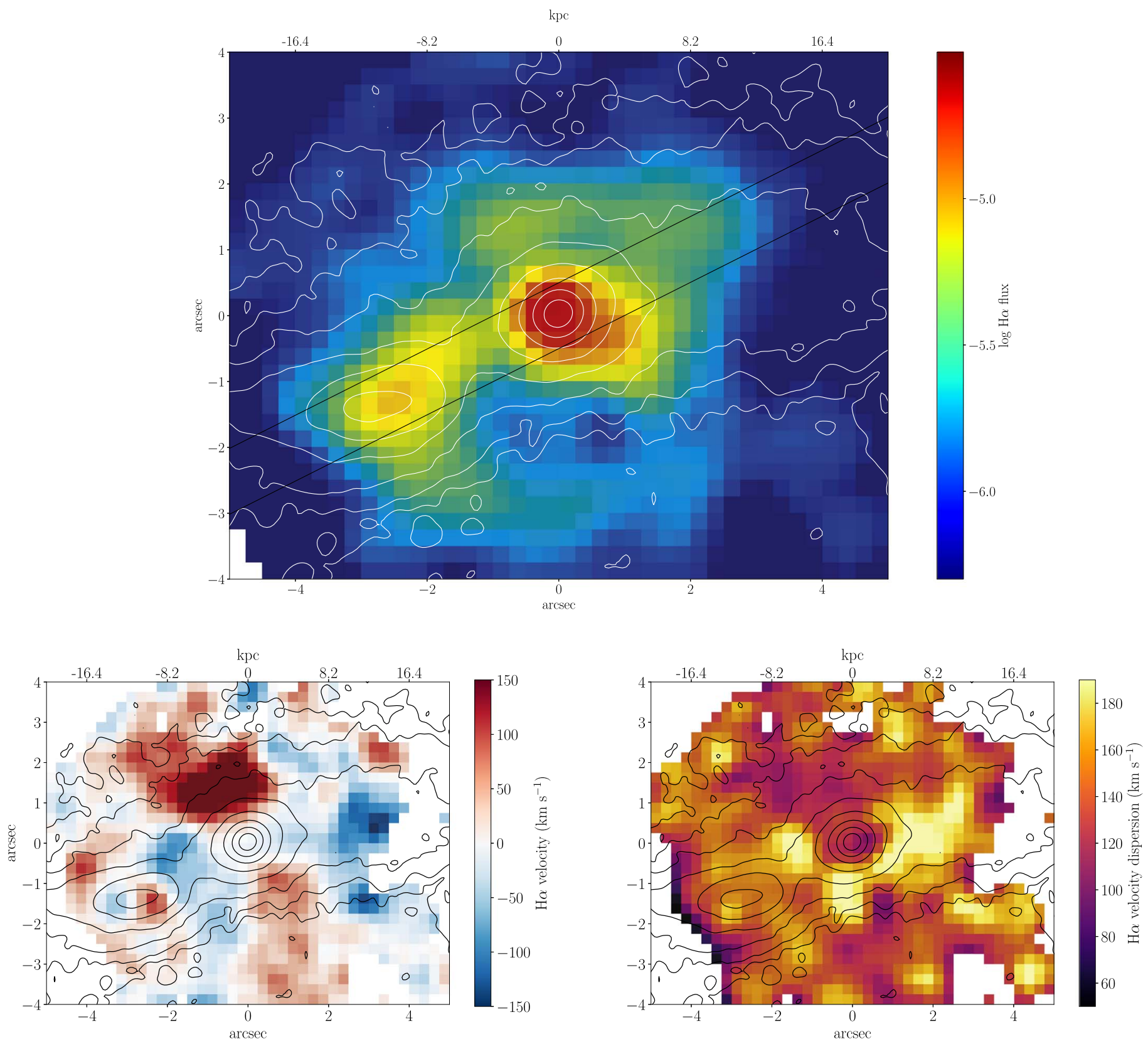

Figure 3. Top: the $\mathrm{H} \alpha$ flux (only the narrow component, relative flux units in logarithmic scale) derived from the IFU MEGARA at GTC. Contours are $J$-band image (Figure 1) logarithmically scaled in intervals of 0.1 dex starting at $3 \sigma$ background. A representation of the slit position for the long-slit spectrographs ISIS at WHT and OSIRIS at GTC is shown with parallel black lines. Bottom: Spatial distribution of the velocity (left) and velocity dispersion (right). We have performed a multiGaussian fitting to the $\mathrm{H} \alpha$ line in the MEGARA IFU data. In this plot, we present the data from the narrow component.

\section{Discussion}

\subsection{Host Galaxy and Merger Environment}

The results acquired from the Subaru imaging and subsequent follow-up observations of TXS 2116-077 confirm its ongoing merger with a Seyfert 2 galaxy. This is probably the first unambiguous detection of a $\gamma$-ray emitting jet hosted by a merging system and hints that relativistic jets can be triggered by galaxy mergers (Chiaberge et al. 2015). Note that a few recent studies have also reported the identification of minor merging events associated with NLSy1 galaxies (e.g., Järvelä et al. 2018; Olguín-Iglesias et al. 2020), however, due to a lack of spectroscopic redshift measurement of the companion, strong claims could not be made. Berton et al. (2019) presented the spectroscopic confirmation of the merger of a radio-loud NLSy1 galaxy IRAS $20181-2244(z=0.185)$, however, this object is yet to be detected in the $\gamma$-ray band and thus its extent of relativistic beaming is uncertain. Furthermore, the small physical separation $(\sim 12 \mathrm{kpc})$ and the presence of AGN activity in both galaxies indicates that this is a merger in the late stage of evolution, i.e., close to coalescence (Di Matteo et al. 2005; Van Wassenhove et al. 2012). Both galaxies are embedded in a common, $70 \mathrm{kpc}$ extended envelope composed of gas and stars, and the companion galaxy exhibits morphological features associated with tidal disruption (Figure 1).

The photometric decomposition of the Subaru image was performed with the $2 \mathrm{D}$ fitting algorithm GALFIT (Peng et al. 


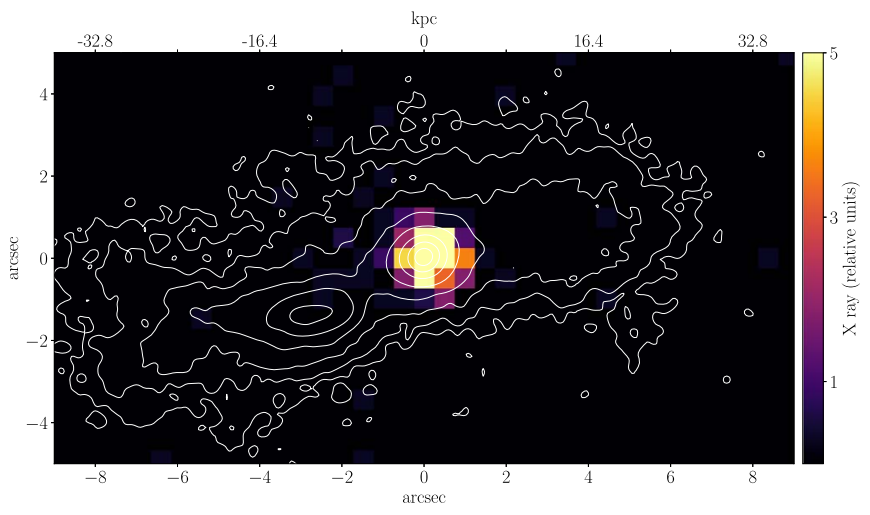

Figure 4. The $0.5-7 \mathrm{keV}$, exposure-corrected Chandra X-ray image of the merging system TXS 2116-077. The resolution of the image is 0 .' 49 per pixel. For a comparison, we overplot the Subaru IR contours as in Figure 3. North is up and east to the left.

2010). The star SDSS J211853.33-073214.3 was used to model the point-spread function (PSF) and remove the AGN contamination. A nearby source-free region was used as the sky background. First, we fitted both galaxies only with the PSF and then added components, mainly Sérsic profiles, based on the leftover emission in the residual image and the reduced $\chi^{2}$ value. Typically, a smaller value of the Sérsic index $(n \lesssim 2)$ is associated with galaxies with disk-like morphology and pseudobulges, and larger values of $n(>2)$ with elliptical galaxies and classical bulges.

The best-fitted model includes two Sérsic functions for each AGN and three Fourier components (see Peng et al. 2010, for details) to take into account the extended emission. The resultant fitting parameters are provided in Table 1 and the results of the fitting are shown in Figure 5. For both $\gamma$-NLSy 1 and the companion, first Sérsic functions with $n=1.48 \pm 0.06$ and $0.73 \pm 0.03$, respectively, resemble that of pseudobulges in late-type galaxies. The physical interpretation of the second Sérsic components is tedious due to disturbed morphologies of both galaxies. However, the small Sérsic index for this component indicates the presence of a disk/bar in both galaxies. Altogether, it can be concluded that both merging galaxies are "young" and host pseudobulges, similar to what is commonly known for other NLSy1 (Mathur 2000; Järvelä et al.

2018; Olguín-Iglesias et al. 2020). This reinforces the hypothesis that $\gamma$-NLSy1 galaxies, and NLSy1s in general, are young sources within the AGN evolution scheme.

The BPT diagram (Baldwin et al. 1981) is a powerful tool to explore the excitation mechanism of the ionized gas. We used the WHT and GTC long-slit spectroscopic observations and extracted spectra at eight locations along the slit with the motivation to understand the physical behavior of the merging environment. In Figure 6, we show the location of seven of the eight spectra extracted ${ }^{13}$ from the long-slit spectroscopy (large red dots) over the background of the BPT diagram of Kewley et al. (2006) for 85,224 galaxies from the SDSS data release 4. Because these seven spectra are extracted from the long-slit along a stretch of $15^{\prime \prime}$ (see black lines in Figure 3), we can firmly state that a large fraction of the gas along the slit in the merging system is affected by AGN and shocks, i.e., physical processes other than due to hot, massive stars. The BPT

\footnotetext{
13 The location of the extracted spectra along the long-slit is shown in the right panel of Figure 7. The spectrum at location 7 in Figure 7 does not have a measurable $\mathrm{H} \beta$ line, hence it is excluded.
}

Table 1

Best-fit GALFIT Modeling Parameters

\begin{tabular}{lrr}
\hline \hline & Function & \\
Parameter & Sérsic 1 & \multicolumn{1}{c}{ Sérsic 2} \\
\hline & $\gamma$-NLSy1 galaxy & \\
Mag & $19.84 \pm 0.01$ & $19.49 \pm 0.01$ \\
$r_{\mathrm{e}}(\mathrm{kpc})$ & $2.35 \pm 0.05$ & $8.94 \pm 0.24$ \\
$n$ & $1.48 \pm 0.06$ & $0.53 \pm 0.01$ \\
Axial ratio & $0.68 \pm 0.01$ & $0.52 \pm 0.01$ \\
PA $\left(^{\circ}\right)$ & $-79.54 \pm 1.25$ & $97.24 \pm 0.51$ \\
\hline & & \\
Mag & $20.03 \pm 0.01$ & $19.91 \pm 0.02$ \\
$r_{\mathrm{e}}(\mathrm{kpc})$ & $3.46 \pm 0.03$ & $8.47 \pm 0.14$ \\
$n$ & $0.73 \pm 0.03$ & $0.71 \pm 0.02$ \\
Axial ratio & $0.44 \pm 0.01$ & $0.53 \pm 0.01$ \\
PA $\left(^{\circ}\right)$ & $-80.48 \pm 0.46$ & $-88.15 \pm 0.79$ \\
\hline
\end{tabular}

Note. $n$ and PA are the Sérsic index and the position angle, respectively. The reduced $\chi^{2}$ of the fit is 1.16 .

diagram was also used to elucidate the nature of the enhanced $\mathrm{H} \alpha$ emitting ring surrounding the $\gamma$-NLSy1 galaxy observed in the MEGARA IFU image (Figure 3, top panel) which confirmed that it is excited by the merger induced shocks and the AGN emission.

After correcting for $3.6 \AA$ instrumental resolution, the full width at half maximum (velocity dispersion) value for the emission line [O III] $5007 \AA$ is $10.29 \AA\left(208 \mathrm{~km} \mathrm{~s}^{-1}\right)$ for the Seyfert 2 nucleus. The FWHM[O III] can be converted to the stellar velocity dispersion $(\sigma)$ by $\sigma=$ FWHM[O III $] / 2.35$ (Nelson \& Whittle 1995; Wang \& Lu 2001). Using the known statistical correlation between the mass of the central black hole and $\sigma$ (Wang \& Lu 2001; Grupe \& Mathur 2004; Gültekin et al. 2009), this value transforms to a black hole mass of $\sim 4 \times 10^{6} M_{\odot}$ for the companion Seyfert 2 galaxy. For the $\gamma-$ NLSy1 galaxy, on the other hand, we used the well calibrated empirical relations (see Shen et al. 2011) adopting the conventional $\mathrm{H} \beta$ FWHM $\left(\sim 1900 \mathrm{~km} \mathrm{~s}^{-1}\right)$ and continuum luminosity at 5100 Angstrom $\left(\sim 10^{44} \mathrm{erg} \mathrm{s}^{-1}\right.$; see also Rakshit et al. 2017). The derived black hole mass is $\sim 3 \times 10^{7} M \odot$, similar to that reported in literature (e.g., Rakshit et al. 2017). Note that the typical uncertainties associated with these approaches are 0.3-0.5 dex (Vestergaard \& Peterson 2006; Gültekin et al. 2009; Shen et al. 2011). Furthermore, since the black hole mass and the host galaxy properties are significantly correlated (Di Matteo et al. 2005; Reines \& Volonteri 2015), the total stellar mass is estimated as $\sim 1 \times 10^{11} M_{\odot}$ and $\sim 2 \times 10^{10} M_{\odot}$ for the $\gamma$-NLSy1 and the companion galaxy, respectively. Based on the large ratio of the stellar masses, the observed event can be classified as a "minor" merger (Lotz et al. 2011).

\subsection{Merger Timescale}

We use the spectral population synthesis code STARLIGHT (Cid Fernandes et al. 2005) to derive key population parameters, such as mean age and metallicity of the merging system. The continuum stellar spectra (masking the emission lines) were fitted for each of the eight spectra extracted from the spatial locations along the OSIRIS and ISIS slits, as demonstrated in the left panel of Figure 7. We retrieved the star formation history, the dust extinction, mass weighted stellar 


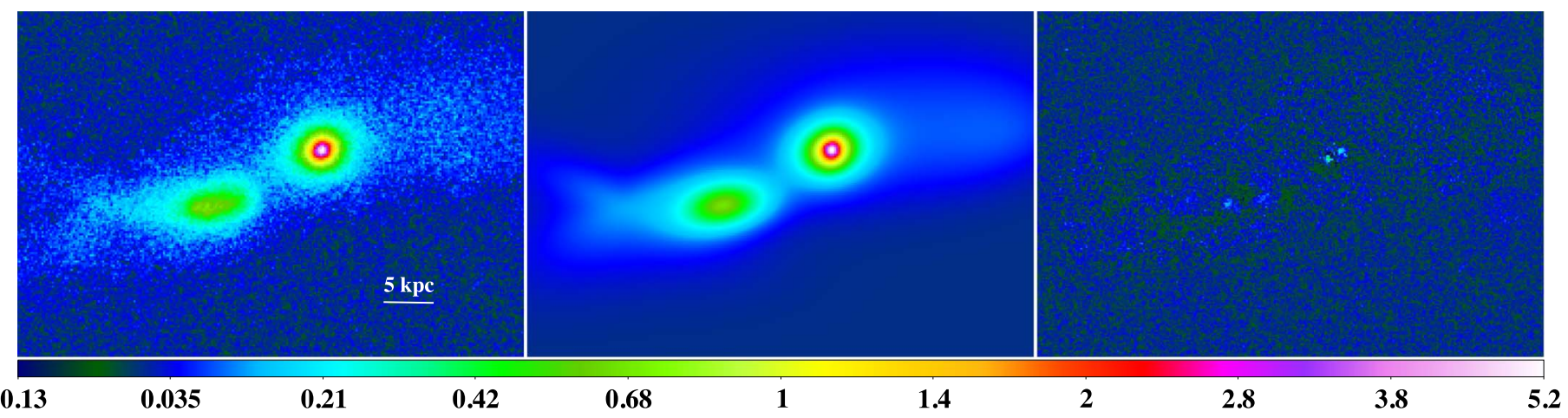

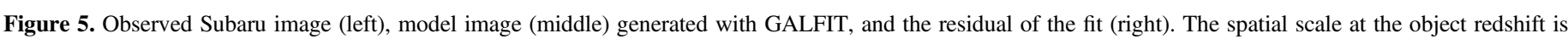
indicated in the left panel. Other information is the same as in Figure 1.

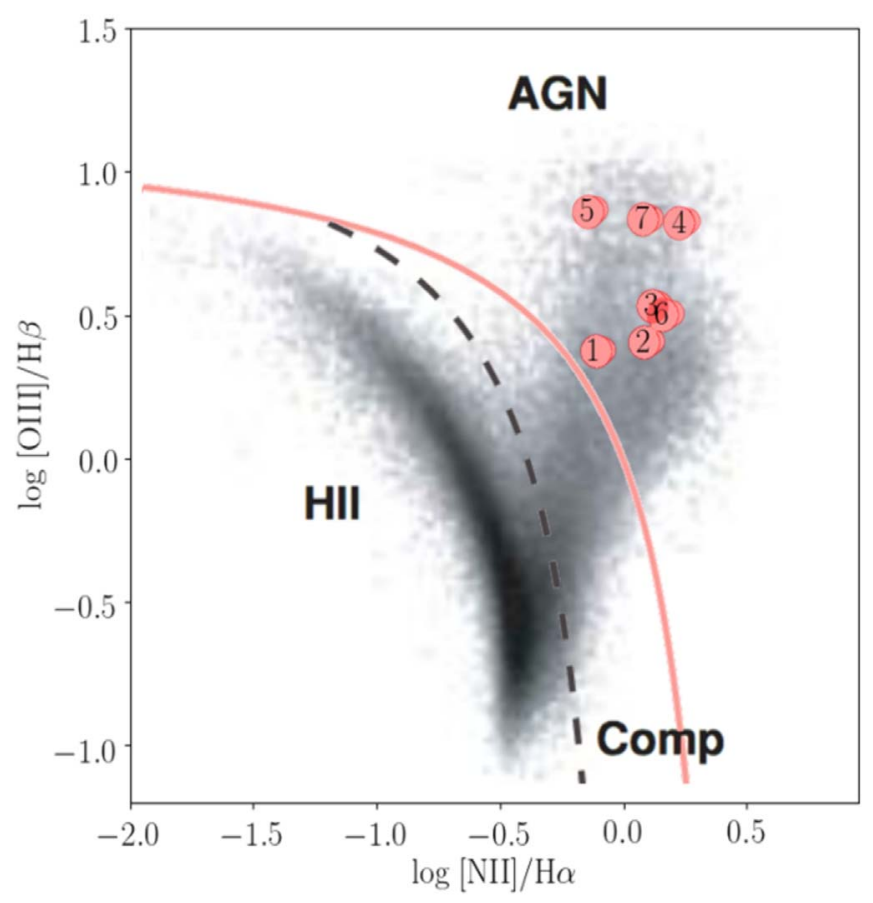

Figure 6. BPT diagram (Baldwin et al. 1981). Large red dots refer to the individual spectra extracted along the long-slit (see Figures 3 and 7). Dots corresponding to $\gamma$-NLSy1 and Seyfert 2 nuclei are labeled "5" and " 4 ", respectively. The gray data points refer to 85,224 galaxies from the SDSS DR4 (Kewley et al. 2006). The region labeled "H II" marks the sequence of ionization from hot massive stars; whereas, that labeled with "AGN" marks ionization by a harder AGN non-stellar continuum or by shocks. The two lines drawn separate the pure stellar from the pure AGN ionization in a "Composite" region. Three upper points clearly indicate AGN ionization, while the other four lower points are in a region where both AGN and shock ionization dominate.

metallicity, and luminosity weighted stellar ages and show them in the right panel of Figure 7 . We used a set of 163 single stellar populations sampling the $3 \mathrm{Myr}$ to $9.75 \mathrm{Gyr}^{14}$ age range with 33 logarithmically spaced age bins and five metallicities between 0.02 and 2.5 solar (S. Charlot and G. Bruzual 2007, private communication), assuming a Chabrier initial mass function. Further details about STARLIGHT can be found on the project web page http://www.starlight.ufsc.br, and a review of stellar populations fitting techniques is available at http:// www.sedfitting.org (Walcher et al. 2011).

\footnotetext{
14 Note that the upper limit to the oldest population possible in this system is given by the redshift of 0.26 , when the universe was 10.7 Gyr old.
}

As can be seen in Figure 7, the results from the stellar population synthesis calculations performed on the GTC and WHT optical spectra show average (luminosity weighted) stellar ages in the range $\sim 0.5-2.5 \mathrm{Gyr}$, older in the outer halo and younger in the inner region around the Seyfert 2 nucleus. The presence of the younger stellar population in proximity to the merging nuclei indicates that the starburst activity has recently begun in the central regions. This result is expected if the spectra are dominated by merger driven activity in the past 0.5-2 Gyr (Moreno et al. 2015). This is because the star formation history that develops along the evolution of a merging pair of galaxies depends on the distance between the galaxies during the different binding passages. For example, Figures 3 and 4 in Moreno et al. (2015) show the star formation evolution from the approach and first passage until the coalescence. Clearly, the window of higher star formation, i.e., when more gas is available to form stars and also to fall into the nuclei, is in the approximate age range $0.5-2 \mathrm{Gyr}$. The detailed history of star formation and nuclear activity also depends on the relative masses and morphology of the two merging galaxies and on the relative angular momenta of the spirals and the merging orbit; but models show that nuclear activity follows after the main bouts of star formation. Indeed, Van Wassenhove et al. (2012) find that strong dual AGN activity occurs during the late phases of the mergers, at small separations $(\lesssim 10 \mathrm{kpc})$. Thus, the results from the analysis of the stellar populations and the nuclear activity in TXS 2116 -077 , especially the merger timescale, match quite precisely the expectations from the numerical simulations.

From the total narrow $\mathrm{H} \alpha$ flux measured in the MEGARA data $(15.7 \mathrm{mJy})$, the star formation rate in the system is 3.8 $M_{\odot} \mathrm{yr}^{-1}$, using the established correlation of star formation rate with $\mathrm{H} \alpha$ emission (Kennicutt 1998). Using the correlation between the global star formation rate and the galaxy stellar mass (González Delgado et al. 2016; Cortijo-Ferrero et al. 2017), this places TXS 2116-077 just within but somewhat high in the star-forming main sequence, implying that the star formation is possibly enhanced by the merging process when compared to normal late-type spiral galaxies (González Delgado et al. 2016).

\subsection{Kinematic Age of the Radio Jet and a Comparison with the Merger Timescale}

Since the radio jet in TXS 2116-077 remains compact down to milliarcsecond scale, we have computed the upper limit to its projected length at $z=0.26$ as $0.02 \mathrm{kpc}$, using the 

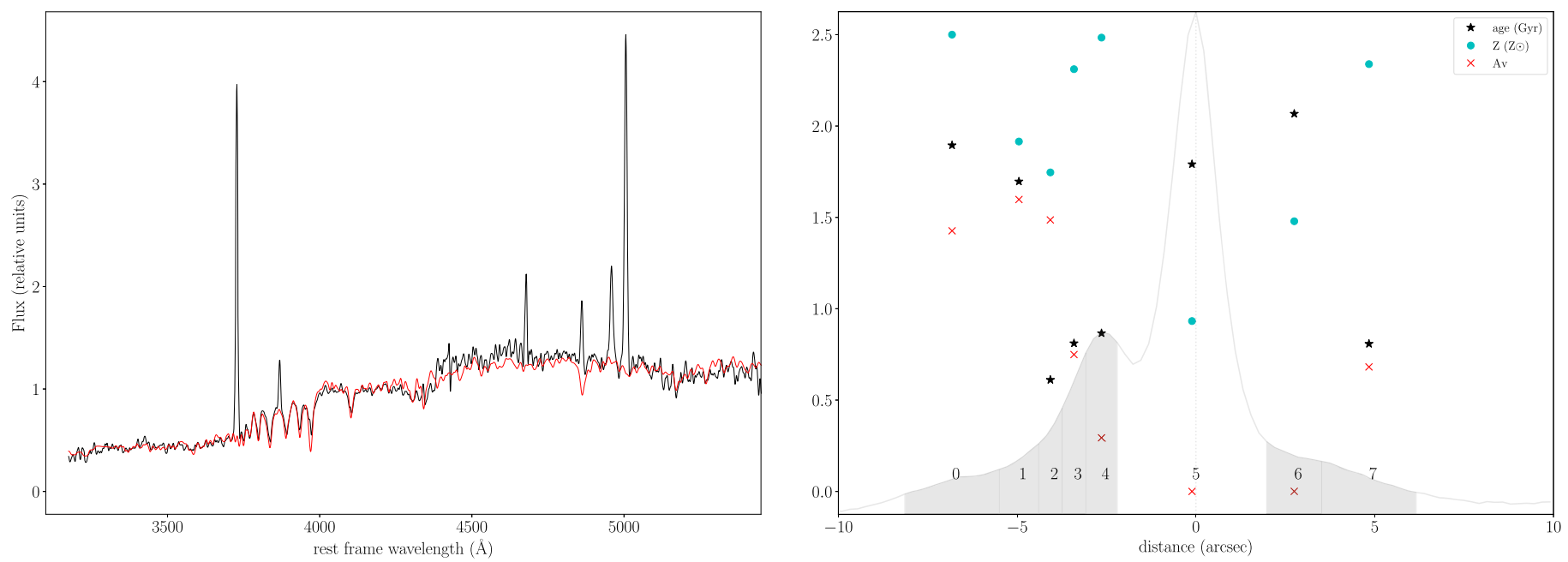

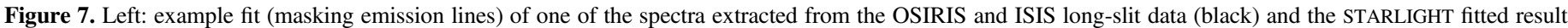

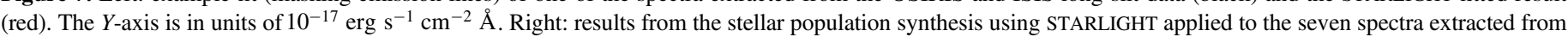

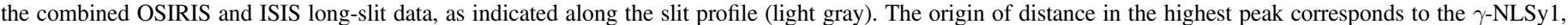

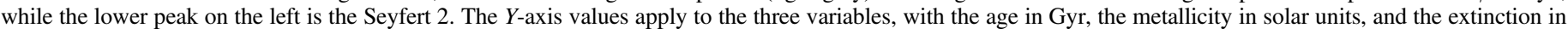

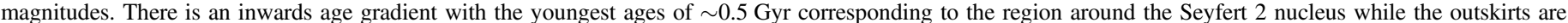

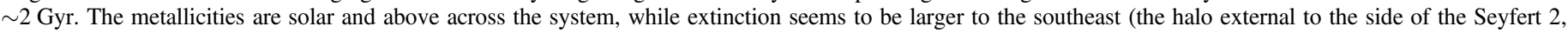
Figure 1).

VLBA beam size of $(5.3 \times 2.4) \mathrm{mas}^{2}$. Considering a viewing angle of $3^{\circ}$, typical for $\gamma$-ray emitting beamed AGNs (Ghisellini et al. 2014; Paliya et al. 2017), the true length turns out to be $<1 \mathrm{kpc}$ after correcting for projection effects. This gives the kinematic age of the jet as $<15 \mathrm{kyr}$, assuming a typical jet velocity of $0.1 c$ (e.g., Gitti et al. 2013). The age will be even shorter for the larger jet velocity/viewing angle. This finding suggests a "young" jet in TXS 2116-077, which is in line with the hypothesis that NLSy1 jets are likely to be in the early phase of evolution (Singh \& Chand 2018). Note that the radio emission in NLSy1 galaxies could be originated from the star formation activities and/or due to nonrelativistic kiloparsec-scale outflows (Gallimore et al. 2006). However, the observation of the flux variability, especially in the $\gamma$-ray band, and also the detection of a flat $\mathrm{GHz}$ frequency spectrum (Yang et al. 2018) indicates that the radio emission detected from TXS 2116-077 is jet dominated and hence the derived kinematic age is reliable.

A comparison of the jet kinematic age $(<15 \mathrm{kyr})$ with the merger timescale $(0.5-2 \mathrm{Gyr})$ suggests the jet to be considerably younger than the merger. This observation provides supportive evidence for a scenario in which relativistic jets are triggered by galaxy mergers. Though high-quality observations, similar to those presented here, are not available for many jetted AGNs, recent observing campaigns focused on the high-resolution host galaxy imaging of radio-loud NLSy1 galaxies are also converging to this scenario (see, e.g., Berton et al. 2019; Olguín-Iglesias et al. 2020). In particular, OlguínIglesias et al. (2020) studied a sample of $\gamma$-NLSy1s and reported that minor mergers could be responsible for the observed nuclear activity in this class of AGN. Though host galaxy observations of a few other sources revealed a rather secular driven growth of the system (Kotilainen et al. 2016) and a few studies also claimed elliptical hosts (e.g., D'Ammando et al. 2017), the majority of the $\gamma$-NLSy1s undoubtedly reside in young, late-type galaxies in which jet launching is likely to be triggered via galaxy mergers. Similar results are found in various studies focusing on more powerful, radio-loud quasars
(Ramos Almeida et al. 2013; Chiaberge et al. 2015; OlguínIglesias et al. 2016). The fact that radio-loud NLSy1s are likely to be living in denser large-scale environments (Järvelä et al. 2017) where mergers could be frequent, also provides supportive evidence for the merger triggered jet launching. Combining this with the idea of the young nature of NLSy1s, it can be concluded that $\gamma$-NLSy1 galaxies represent the beginning phase of the jet activity.

There is significant support of the idea that rotating supermassive black holes produce jets and their origin is connected to magnetic fields generated in accretion disks of spinning black holes (Blandford \& Znajek 1977; Meier 1999). Recent 3D relativistic magnetohydrodynamic simulations support this spin paradigm (see, e.g., Tchekhovskoy et al. 2011) and demonstrate that prograde disk accretion onto a rapidly spinning black hole most efficiently generates jets. Combining this with the idea that galaxy mergers trigger AGNs (Di Matteo et al. 2005; Hopkins \& Quataert 2011) leads to the expectation that a merger may produce the needed high spin. Galaxy mergers can efficiently drive gas inflows all the way to the central regions of the galaxy and all the way to the accretion disk that feeds the central black hole.

\section{Summary}

We have carried out a comprehensive multifrequency campaign to study the host galaxy properties of a $\gamma$-NLSy 1 galaxy, TXS 2116-077. The high-resolution imaging done with the Subaru telescope has revealed the $\gamma$-NLSy1 to be merging with a nearby $(\sim 12 \mathrm{kpc})$ galaxy. Subsequent optical spectroscopic observations from GTC and WHT have confirmed the merging phenomenon and revealed that both galaxies host AGNs at their centers with the companion galaxy being a Seyfert 2. Furthermore, IFU observations from MEGARA, along with the long-slit spectroscopy with ISIS at WHT and OSIRIS at GTC, have provided a very rare view of the merging environment surrounding the relativistic jet. We have also found that TXS 2116-077 hosts a compact radio jet whose kinematic age is considerably smaller than the merger 
timescale derived from the stellar population synthesis. Our findings are aligned with other recent studies focused on the host galaxy imaging of NLSy1s (Olguín-Iglesias et al. 2020) and support a scenario in which relativistic jets are triggered by galaxy mergers (Chiaberge et al. 2015).

Black holes in NLSy1 galaxies usually grow via secular processes, develop pseudobulges, mostly remain radio-quiet, and evolve to broad-line Seyfert $1 \mathrm{~s}$ (Mathur 2000). Those residing in dense large-scale environments can frequently undergo mergers (Chiaberge et al. 2015; Järvelä et al. 2017), triggering rapid black hole growth due to inflow of gas driven by gravitational torques (Barnes \& Hernquist 1991, 1996), and possibly spin-up the black hole leading to the launch of the relativistic jet. In this evolutionary scenario, $\gamma$-NLSy1s can be considered as nascent blazars and as such they are the best jetted systems to explore the jet launching regions and their associated physical processes.

Thanks are due to the journal referee for constructive criticism. V.S.P. and M.A. acknowledge the prompt Chandra DDT observation of TXS 2116-077 and funding under contract DD8-19104X. V.S.P. is grateful to C. Y. Peng for his assistance with the GALFIT modeling. M.A. acknowledges discussions with R. Blandford and C. Conselice and the support of NSF through grant AST-1715256 F.P. acknowledges support from the Spanish MICINN grant PGC2018101931-B-100. E.P. and R.G.B. acknowledge financial support from the Spanish Ministry of Economy and Competitiveness through grant AYA2016-77846-P. E.P., R.G.B., and F.P. acknowledge support from the State Agency for Research of the Spanish MCIU through the "Center of Excellence Severo Ochoa" award to the Instituto de Astrofísica de Andalucía (SEV-2017-0709). A.D. acknowledges the support of the Ramón y Cajal program from the Spanish MINECO. This work is based on data collected at the Subaru Telescope, which is operated by the National Astronomical Observatory of Japan. Based on service observations made with the $4.2 \mathrm{~m}$ WHT operated by the Isaac Newton Group of Telescopes and installed in the Spanish Observatorio del Roque de los Muchachos of the Instituto de Astrofísica de Canarias, in the island of La Palma. We are grateful to staff astronomers Antonio Cabrera at GTC and Ian Skillen at WHT for carrying out MEGARA, OSIRIS, and ISIS observations. This work is based on observations made with the GTC, in the Spanish Observatorio del Roque de los Muchachos of the Instituto de Astrofísica de Canarias, under Director's Discretionary Time.

Facilities: Subaru, GTC, WHT, Chandra, VLA.

Software: IRAF (Tody 1986, 1993), MEGARA data reduction pipeline (S. Pascual 2020, in preparation), AIPS (van Moorsel et al. 1996), CIAO (Fruscione et al. 2006), XSPEC (Arnaud 1996), starlight (Cid Fernandes et al. 2005), GALFIT (Peng et al. 2010).

\section{ORCID iDs}

Vaidehi S. Paliya (1) https://orcid.org/0000-0001-7774-5308 Enrique Pérez (i) https://orcid.org/0000-0001-9737-4559 Rubén García-Benito (i) https://orcid.org/0000-00027077-308X Marco Ajello (i) https://orcid.org/0000-0002-6584-1703 Antxon Alberdi (1) https://orcid.org/0000-0002-9371-1033 Hyewon Suh (1) https://orcid.org/0000-0002-2536-1633 Alberto Domínguez (i) https://orcid.org/0000-0002-3433-4610
Stefano Marchesi (i) https://orcid.org/0000-0001-5544-0749

Dieter Hartmann (ib https://orcid.org/0000-0002-8028-0991

Marco Chiaberge 나 https://orcid.org/0000-0003-1564-3802

\section{References}

Abdo, A. A., Ackermann, M., Ajello, M., et al. 2009, ApJL, 707, L142 Arnaud, K. A. 1996, in ASP Conf. Ser. 101, Astronomical Data Analysis Software and Systems V, ed. G. H. Jacoby \& J. Barnes (San Francisco, CA: ASP), 17

Baldwin, J. A., Phillips, M. M., \& Terlevich, R. 1981, PASP, 93, 5

Barnes, J. E., \& Hernquist, L. 1996, ApJ, 471, 115

Barnes, J. E., \& Hernquist, L. E. 1991, ApJL, 370, L65

Berton, M., Congiu, E., Ciroi, S., et al. 2019, AJ, 157, 48

Berton, M., Congiu, E., Järvelä, E., et al. 2018, A\&A, 614, A87

Blandford, R. D., \& Znajek, R. L. 1977, MNRAS, 179, 433

Boroson, T. A., \& Green, R. F. 1992, ApJS, 80, 109

Cash, W. 1979, ApJ, 228, 939

Chiaberge, M., Gilli, R., Lotz, J. M., \& Norman, C. 2015, ApJ, 806, 147

Chiaberge, M., \& Marconi, A. 2011, MNRAS, 416, 917

Cid Fernandes, R., Mateus, A., Sodré, L., Stasińska, G., \& Gomes, J. M. 2005, MNRAS, 358, 363

Cortijo-Ferrero, C., González Delgado, R. M., Pérez, E., et al. 2017, A\&A, 607, A70

D’Ammando, F., Acosta-Pulido, J. A., Capetti, A., et al. 2017, MNRAS, 469, L11

Di Matteo, T., Springel, V., \& Hernquist, L. 2005, Natur, 433, 604

Fabian, A. C. 2012, ARA\&A, 50, 455

Floyd, D. J. E., Axon, D., Baum, S., et al. 2008, ApJS, 177, 148

Foschini, L., Berton, M., Caccianiga, A., et al. 2015, A\&A, 575, A13

Fruscione, A., McDowell, J. C., Allen, G. E., et al. 2006, Proc. SPIE, 6270, $62701 \mathrm{~V}$

Gallimore, J. F., Axon, D. J., O'Dea, C. P., Baum, S. A., \& Pedlar, A. 2006, AJ, 132, 546

Ghisellini, G., Tavecchio, F., Maraschi, L., Celotti, A., \& Sbarrato, T. 2014, Natur, 515,376

Gil de Paz, A., Carrasco, E., Gallego, J., et al. 2016, Proc. SPIE, 9908, 99081K

Gitti, M., Giroletti, M., Giovannini, G., Feretti, L., \& Liuzzo, E. 2013, A\&A, 557, L14

González Delgado, R. M., Cid Fernandes, R., Pérez, E., et al. 2016, A\&A, 590, A44

Goodrich, R. W. 1989, ApJ, 342, 224

Grupe, D., \& Mathur, S. 2004, ApJL, 606, L41

Gültekin, K., Richstone, D. O., Gebhardt, K., et al. 2009, ApJ, 698, 198

Hayano, Y., Takami, H., Oya, S., et al. 2010, Proc. SPIE, 7736, 77360N

Hopkins, P. F., \& Elvis, M. 2010, MNRAS, 401, 7

Hopkins, P. F., \& Quataert, E. 2011, MNRAS, 415, 1027

Järvelä, E., Lähteenmäki, A., \& Berton, M. 2018, A\&A, 619, A69

Järvelä, E., Lähteenmäki, A., Lietzen, H., et al. 2017, A\&A, 606, A9

Kalberla, P. M. W., Burton, W. B., Hartmann, D., et al. 2005, A\&A, 440, 775

Kellermann, K. I., Sramek, R., Schmidt, M., Shaffer, D. B., \& Green, R. 1989, AJ, 98, 1195

Kennicutt, R. C., Jr. 1998, ARA\&A, 36, 189

Kewley, L. J., Groves, B., Kauffmann, G., \& Heckman, T. 2006, MNRAS, 372,961

Kobayashi, N., Tokunaga, A. T., Terada, H., et al. 2000, Proc. SPIE, 4008, 1056

Kormendy, J., \& Richstone, D. 1995, ARA\&A, 33, 581

Kotilainen, J. K., Falomo, R., \& Scarpa, R. 1998, A\&A, 336, 479

Kotilainen, J. K., León-Tavares, J., Olguín-Iglesias, A., et al. 2016, ApJ, 832,157

Laor, A. 2000, ApJL, 543, L111

Lotz, J. M., Jonsson, P., Cox, T. J., et al. 2011, ApJ, 742, 103

Mathur, S. 2000, MNRAS, 314, L17

Meier, D. L. 1999, ApJ, 522, 753

Moreno, J., Torrey, P., Ellison, S. L., et al. 2015, MNRAS, 448, 1107

Nelson, C. H., \& Whittle, M. 1995, ApJS, 99, 67

Nilsson, K., Pursimo, T., Heidt, J., et al. 2003, A\&A, 400, 95

Olguín-Iglesias, A., Kotilainen, J., \& Chavushyan, V. 2020, MNRAS, 492,1450

Olguín-Iglesias, A., León-Tavares, J., Kotilainen, J. K., et al. 2016, MNRAS, 460, 3202

Osterbrock, D. E., \& Pogge, R. W. 1985, ApJ, 297, 166

Paliya, V. S., Ajello, M., Rakshit, S., et al. 2018, ApJL, 853, L2

Paliya, V. S., Koss, M., Trakhtenbrot, B., et al. 2019a, ApJ, 881, 154 
Paliya, V. S., Marcotulli, L., Ajello, M., et al. 2017, ApJ, 851, 33

Paliya, V. S., Parker, M. L., Jiang, J., et al. 2019b, ApJ, 872, 169

Peng, C. Y., Ho, L. C., Impey, C. D., \& Rix, H.-W. 2010, AJ, 139, 2097

Planck Collaboration, Ade, P. A. R., Aghanim, N., et al. 2016, A\&A, 594, A13

Rakshit, S., Stalin, C. S., Chand, H., \& Zhang, X.-G. 2017, ApJS, 229, 39

Ramos Almeida, C., Bessiere, P. S., Tadhunter, C. N., et al. 2013, MNRAS, 436, 997

Reines, A. E., \& Volonteri, M. 2015, ApJ, 813, 82

Shen, Y., Richards, G. T., Strauss, M. A., et al. 2011, ApJS, 194, 45

Singh, V., \& Chand, H. 2018, MNRAS, 480, 1796

Tchekhovskoy, A., Narayan, R., \& McKinney, J. C. 2011, MNRAS, 418, L79

Tody, D. 1986, Proc. SPIE, 627, 733
Tody, D. 1993, in ASP Conf. Ser. 52, IRAF in the Nineties, ed. R. J. Hanisch, R. J. V. Brissenden, \& J. Barnes (San Francisco, CA: ASP), 173

Toomre, A., \& Toomre, J. 1972, ApJ, 178, 623

van Moorsel, G., Kemball, A., \& Greisen, E. 1996, in ASP Conf. Ser. 101, AIPS Developments in the Nineties, ed. G. H. Jacoby \& J. Barnes (San Francisco, CA: ASP), 37

Van Wassenhove, S., Volonteri, M., Mayer, L., et al. 2012, ApJL, 748, L7 Vestergaard, M., \& Peterson, B. M. 2006, ApJ, 641, 689

Walcher, J., Groves, B., Budavári, T., \& Dale, D. 2011, Ap\&SS, 331, 1

Wang, T., \& Lu, Y. 2001, A\&A, 377, 52

Wilson, A. S., \& Colbert, E. J. M. 1995, ApJ, 438, 62

Yang, H., Yuan, W., Yao, S., et al. 2018, MNRAS, 477, 5127 